PAPER • OPEN ACCESS

\title{
On Hölder solutions to the spiral winding problem
}

To cite this article: Jonathan M Fraser 2021 Nonlinearity 343251

View the article online for updates and enhancements. 


\title{
On Hölder solutions to the spiral winding problem
}

\author{
Jonathan M Fraser (1) \\ School of Mathematics and Statistics, The University of St Andrews, St Andrews, \\ KY16 9SS, United Kingdom \\ E-mail: jmf32@st-andrews.ac.uk
}

Received 10 March 2020, revised 26 January 2021

Accepted for publication 17 February 2021

Published 7 May 2021

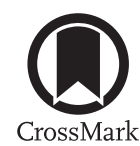

\begin{abstract}
The winding problem concerns understanding the regularity of functions which map a line segment onto a spiral. This problem has relevance in fluid dynamics and conformal welding theory, where spirals arise naturally. Here we interpret 'regularity' in terms of Hölder exponents and establish sharp results for spirals with polynomial winding rates, observing that the sharp Hölder exponent of the forward map and its inverse satisfy a formula reminiscent of Sobolev conjugates. We also investigate the dimension theory of these spirals, in particular, the Assouad dimension, Assouad spectrum and box dimensions. The aim here is to compare the bounds on the Hölder exponents in the winding problem coming directly from knowledge of dimension (and how dimension distorts under Hölder image) with the sharp results. We find that the Assouad spectrum provides the best information, but that even this is not sharp. We also find that the Assouad spectrum is the only 'dimension' which distinguishes between spirals with different polynomial winding rates in the superlinear regime.
\end{abstract}

Keywords: spiral, winding problem, Holder exponents, Assouad dimension, box dimension, Assouad spectrum

Mathematics Subject Classification numbers: 28A80, 26A16, 37C45, 37C10, 28A78, 34C05.

\section{Introduction: spirals and the winding problem}

Spirals appear naturally across mathematics and wider science, often arising via a dynamical system or geometric constraint. One of the simplest examples is the Archimedean spiral, which is the trajectory of a point moving away from its initial position with constant speed along a line

Recommended by Dr Mark F Demers

Original content from this work may be used under the terms of the Creative Commons Attribution 3.0 licence. Any further distribution of this work must maintain attribution to the author(s) and the title of the work, journal citation and DOI. 

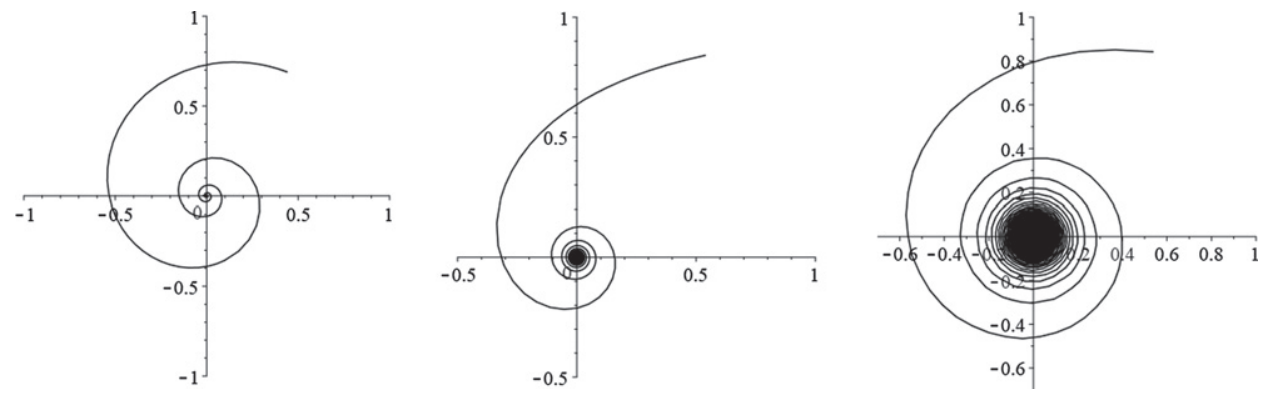

Figure 1. Three spirals: on the left, the logarithmic spiral with $\phi(x)=\exp (-x / 5)$, in the centre a hyperbolic spiral with $\phi(x)=x^{-1}$, and on the right a Lituus with $\phi(x)=x^{-1 / 2}$.

which rotates with constant speed. In fluid dynamics spiral trajectories arise in various models of fluid turbulence and vortex formation. For example, in the well-studied $\alpha$-models for fluid turbulence polynomial spirals appear as the evolution of the half-line $[0, \infty) \subset \mathbb{R}^{2}$ under the resulting two-dimensional flow and the polynomial winding rate depends on the parameter $\alpha$, see [FHT]. See [M, Mo, V, VH] for more specific examples of spirals appearing in fluid turbulence and [T, ZZ] for other dynamical examples giving rise to spiral trajectories where particular attention is paid to dimension.

In a different direction, spirals arise as solutions to various geometric problems. For example, the Lituus is the locus of points $z \in \mathbb{C}$ preserving the area of the circular sector $\{w: \arg (w) \in(0, \arg (z)),|w| \leqslant|z|\}$ (including multiplicity when $\arg (z)>2 \pi)$. Consider also the hyperbolic spiral which is the 'inverse' of the Archimedean spiral. A more sophisticated setting where spirals have proved important is in the theory of conformal welding. This considers the regularity of the induced self-homeomorphism of an oriented Jordan curve arising by composing a Jordan mapping on the interior with the inverse of a Jordan mapping on the exterior. Jordan curves defined using logarithmic spirals (see below) were shown in [KNS] to exhibit an interesting intermediate phenomenon (non-differentiable, but Lipschitz) not previously observed.

Wherever spirals arise, be it via a dynamical system or as the solution to a geometric problem, the form and regularity of the spiral holds relevance for the underlying model or problem. Of course there are many ways to quantify regularity, for example, via various notions of fractal dimension since infinitely wound spirals can be viewed as fractals. Here we consider the winding problem, which characterizes the regularity of a spiral by the regularity of homeomorphisms mapping a line segment to the spiral—such a function is a solution to the winding problem, since it performs the task of winding the line segment to the spiral. A common formulation of this problem is to ask whether or not bi-Lipschitz solutions exist, see [FP, KNS]. Here we search for bi-Hölder solutions in situations where bi-Lipschitz solutions do not exist. This approach appears to be novel and has the advantage of applying to a larger class of spirals. In particular, spirals arising in nature via a dynamical process tend to have polynomial winding rates, which do not admit bi-Lipschitz solutions. Moreover, the Hölder version of the problem is more flexible since, once Hölder solutions are known to exist, one can consider the more refined problem of optimising the Hölder exponents of the solution.

Given a winding function $\phi:[1, \infty) \rightarrow(0, \infty)$, which we assume is continuous, strictly decreasing, and satisfies $\phi(x) \rightarrow 0$ as $x \rightarrow \infty$, the associated spiral is the set (see figure 1)

$$
\mathcal{S}(\phi)=\{\phi(x) \exp (\mathrm{i} x): 1<x<\infty\} \subset \mathbb{C} .
$$


The winding problem concerns the regularity of $\mathcal{S}(\phi)$ by asking how little distortion is required to map $(0,1)$ onto $\mathcal{S}(\phi)$. A well-known and important example of this is that when $\phi(x)=\mathrm{e}^{-c x}$ for some $c>0$, it is possible to map $(0,1)$ onto the so-called logarithmic spiral $\mathcal{S}(\phi)$ via a bi-Lipschitz map. This was first established by Katznelson et al [KNS]. Moreover, if $\phi$ is sub-exponential, that is, if

$$
\frac{\log \phi(x)}{x} \rightarrow 0 \quad(x \rightarrow \infty)
$$

then this cannot be done, thus illustrating that the logarithmic family is sharp for the biLipschitz problem. See Fish and Paunescu [FP] for an elegant proof of this latter fact. In this paper, we wish to understand the sub-exponential regime by considering the Hölder analogue of this problem. Namely, given a sub-exponential winding function, is it possible to map $(0,1)$ onto $\mathcal{S}(\phi)$ via a Hölder map and if so what is the optimal Hölder exponent? It turns out that it is natural to consider bi-Hölder maps (Hölder maps with Hölder inverses), and the interplay between the two Hölder exponents is crucial. Indeed, if one wants to achieve the sharp Hölder exponent for a homeomorphism mapping $(0,1)$ to $\mathcal{S}(\phi)$, then one must sacrifice the Hölder exponent of its inverse and vice versa. This is perhaps surprising since spirals are 'more complex' than $(0,1)$ and therefore one might naively expect that only the Hölder exponent of the forward map is relevant, with the inverse map Lipschitz for most reasonable homeomorphisms.

Recall that, given $\alpha \in(0,1]$ and $X \subset \mathbb{C}$, a function $f: X \rightarrow \mathbb{C}$ is $\alpha$-Hölder if there exists a constant $C>0$ such that, for all $x, y \in X$,

$$
|f(x)-f(y)| \leqslant C|x-y|^{\alpha} .
$$

In the special case when $\alpha=1$, the map is called Lipschitz. Moreover, given $0<\alpha \leqslant 1 \leqslant$ $\beta<\infty$ we say that a homeomorphism $f: X \rightarrow Y$ is $(\alpha, \beta)$-Hölder if there exists a constant $C>0$ such that, for all $x, y \in X$,

$$
C^{-1}|x-y|^{\beta} \leqslant|f(x)-f(y)| \leqslant C|x-y|^{\alpha},
$$

that is, $f$ is $\alpha$-Hölder and $f^{-1}: Y \rightarrow X$ is $\beta^{-1}$-Hölder. Similar to above, $(1,1)$-Hölder maps are called bi-Lipschitz.

In order to simply our exposition we consider a particular family of sub-exponential spirals, namely the polynomial family $\phi_{p}$ defined by $\phi_{p}(x)=x^{-p}$ for $p>0$. Our results apply more generally, but we delay discussion of this until section 6. In fact the spirals associated with $\phi_{p}$ are bi-Lipschitz equivalent to any spiral whose winding function is 'comparable' to $\phi_{p}$, see section 6, and thus behave in exactly the same way in the context of the winding problem. We write $\mathcal{S}_{p}$ for the spiral associated to $\phi_{p}$, that is, we write $\mathcal{S}_{p}=\mathcal{S}\left(\phi_{p}\right)$ for brevity (see figure 1). The spirals $\mathcal{S}_{p}$ are sometimes referred to as generalized hyperbolic spirals (the hyperbolic spiral corresponds to $p=1$ ) and are typically found in nature whenever there is an underlying dynamical process. In contrast, when spirals form in nature in a static setting, they tend to be logarithmic, that is, have exponential winding functions.

A well-studied and important problem in the dimension theory of fractals is to consider how Hölder maps affect a given notion of fractal dimension, see [F]. More precisely, given a notion of dimension, such as the Hausdorff or box dimension, one can often relate the dimensions of $f(K)$ and $K$ for all sets $K$ and Hölder maps $f$ in terms of the Hölder exponents of $f$. As such, knowledge of the dimensions of $\mathcal{S}_{p}$ give rise to bounds on the possible Hölder exponents in the winding problem. In section 4 we consider this problem thoroughly by considering a number of available notions of dimensions. In particular, we consider the Hausdorff, box, and Assouad 
dimensions of the spirals $\mathcal{S}_{p}$ for $p>0$, as well as the Assouad spectrum, which interpolates between the box and Assouad dimensions. We prove that the Assouad spectrum 'separates this class', that is, the Assouad spectra depends on $p$ for all $p>0$, whereas, the Hausdorff, box, and Assouad dimensions fail to do this. We establish precisely how much information can be extracted from dimension theory in the context of the Hölder version of the winding problem, proving that the best information comes from the Assouad spectrum, but even this is not sharp.

Motivated by the above, we propose a general programme of research. Given two bounded homeomorphic sets $X, Y \subset \mathbb{R}^{d}$, first consider the Hölder mapping problem which asks for sharp estimates on $\alpha$ and $\beta$ such that there exists an $(\alpha, \beta)$-Hölder map $f$ with $f(X)=Y$. Secondly, consider the estimates on $\alpha, \beta$ which come directly from knowledge of the dimensions of $X$ and $Y$. The problem is then to determine in which situations the information provided by the dimensions is sharp and when it is not, as well as determining which notion of dimension 'performs best' in a given setting. In particular, the polynomial spirals we consider here are examples where sharp information is not provided by dimension theory, but where the Assouad spectrum performs the best.

\section{Main results: Hölder solutions to the winding problem}

We write $X \lesssim Y$ to mean that $X \leqslant c Y$ for some universal constant $c>0$. We also write $X \gtrsim Y$ to mean $Y \lesssim X$ and $X \approx Y$ to mean that both $X \lesssim Y$ and $X \gtrsim Y$ hold. We write $|E|$ for the diameter of a set $E$. For real numbers $x, y$ we write $x \wedge y=\min \{x, y\}$ and $x \vee y=\max \{x, y\}$.

A useful trick which we will use throughout is to decompose $\mathcal{S}_{p}$ into the disjoint union of 'full turns'

$$
\mathcal{S}_{p}=\bigcup_{k \geqslant 1} \mathcal{S}_{p}^{k}
$$

where

$$
\mathcal{S}_{p}^{k}=\left\{x^{-p} \exp (\mathrm{i} x): 1+2 \pi(k-1)<x \leqslant 1+2 \pi k\right\}
$$

for integer $k \geqslant 1$. Also, given a homeomorphism $f:(0,1) \rightarrow \mathcal{S}_{p}$, we decompose $(0,1)$ into the corresponding half-open intervals

$$
\mathcal{I}^{k}=f^{-1}\left(\mathcal{S}_{p}^{k}\right)
$$

Our first result brings in the interplay between the two Hölder exponents when one considers bi-Hölder functions. It also provides a simple upper bound for the forward Hölder exponent, $\alpha$, considered in isolation. The inverse Hölder exponent, $\beta$, is trivially bounded below by 1 and this cannot be improved without considering $\alpha$.

Theorem 2.1. If $f:(0,1) \rightarrow \mathcal{S}_{p}$ is an $\alpha$-Hölder homeomorphism, then $\alpha<p$. Moreover, if $f:(0,1) \rightarrow \mathcal{S}_{p}$ is an $(\alpha, \beta)$-Hölder homeomorphism, then

$$
\beta \geqslant \frac{p \alpha}{p-\alpha}
$$

Proof. In the first instance suppose only that $f:(0,1) \rightarrow \mathcal{S}_{p}$ is $\alpha$-Hölder. We have

$$
k^{-p} \approx\left|\mathcal{S}_{p}^{k}\right|=\left|f\left(\mathcal{I}^{k}\right)\right| \lesssim\left|\mathcal{I}^{k}\right|^{\alpha}
$$



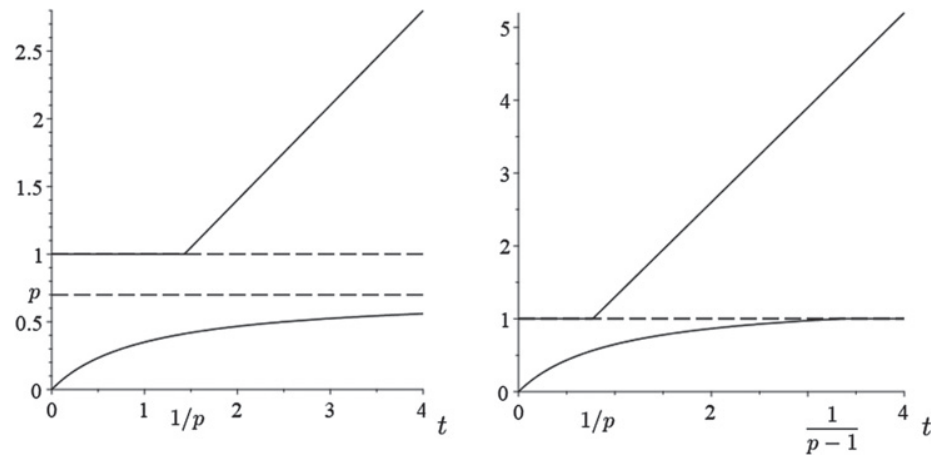

Figure 2. Plots of the sharp Hölder exponents $\alpha, \beta$ for the map $g_{t}$ as functions of $t$ (solid lines). Plots of 1 and $\min (1, p)$ are shown as dashed lines for reference. On the left $p=0.7$ and on the right $p=1.3$. The optimal $\beta$ is achieved for $t \leqslant 1 / p$. Whereas, if $p \leqslant 1$, then the optimal $\alpha$ is only obtained asymptotically as $t \rightarrow \infty$ and, if $p>1$, then the optimal $\alpha$ is obtained for $t \geqslant 1 /(p-1)$.

and therefore

$$
1=\sum_{k=1}^{\infty}\left|\mathcal{I}^{k}\right| \gtrsim \sum_{k=1}^{\infty} k^{-p / \alpha}
$$

which forces $\alpha<p$.

Now suppose $f:(0,1) \rightarrow \mathcal{S}_{p}$ is an $(\alpha, \beta)$-Hölder homeomorphism, which we may assume satisfies $f(x) \rightarrow 0$ as $x \rightarrow 0$ and for convenience we extend $f$ continuously to $[0,1]$. This extension preserves the Hölder property. By the above, we know $p / \alpha>1$. For integer $l \geqslant 1$, let

$$
x_{l}=\sum_{k=l}^{\infty}\left|\mathcal{I}^{k}\right|
$$

where $\mathcal{I}^{k}$ is as in (2.2). Note that $x_{l}$ is the right endpoint of $\mathcal{I}^{l}$. Combining this with (2.3) yields

$$
1 \lesssim \frac{\left|f\left(x_{l}\right)-f(0)\right|}{\left|x_{l}\right|^{\beta}} \lesssim \frac{l^{-p}}{\left(\sum_{k=l}^{\infty}\left|\mathcal{I}^{k}\right|\right)^{\beta}} \lesssim \frac{l^{-p}}{\left(\sum_{k=l}^{\infty} k^{-p / \alpha}\right)^{\beta}} \lesssim \frac{l^{-p}}{l^{(1-p / \alpha) \beta}} \rightarrow 0
$$

as $l \rightarrow \infty$, if $-p-(1-p / \alpha) \beta<0$. This forces

$$
\beta \geqslant \frac{p \alpha}{p-\alpha}
$$

as required.

Despite how simple the proof of theorem 2.1 is, it turns out to be sharp. Moreover, there is a particularly natural family of examples demonstrating this sharpness, which we introduce now (see figure 2). Given $t>0$, define $g_{t}:(0,1) \rightarrow \mathcal{S}_{p}$ by

$$
g_{t}(x)=x^{t p} \exp \left(\mathrm{i} / x^{t}\right)
$$


noting that each $g_{t}$ is clearly a homeomorphism between $(0,1)$ and $\mathcal{S}_{p}$.

Theorem 2.2. For all $p, t>0$, the map $g_{t}$ is a

$$
\left(\frac{t p}{t+1} \wedge 1, t p \vee 1\right)-\text { Hölder. }
$$

homeomorphism between $(0,1)$ and $\mathcal{S}_{p}$, and these Hölder exponents are sharp.

We delay the proof of theorem 2.2 until section 3. It follows immediately from theorem 2.2 that theorem 2.1 is sharp. We provide an alternative direct proof of this in section 5 . Note we only consider $\alpha \geqslant p /(p+1)$ since $\beta$ can be chosen to equal 1 for $\alpha=p /(p+1)$ and so there is no need to consider weaker conditions on $\alpha$.

Corollary 2.3. For $p>0$ and $\alpha \in\left[\frac{p}{p+1}, p\right) \cap(0,1]$, there exists an $\left(\alpha, \frac{p \alpha}{p-\alpha}\right)$-Hölder homeomorphism between $(0,1)$ and $\mathcal{S}_{p}$.

Proof. Fix $p>0$ and $\alpha \in\left[\frac{p}{p+1}, p\right) \cap(0,1]$, consider the map $g_{t}$ with

$$
t=\frac{\alpha}{p-\alpha}
$$

and apply theorem 2.2.

We remark that the sharp relationship between $\alpha$ and $\beta$ given in theorem 2.1 and corollary 2.3 resembles that of Sobolev conjugates. In particular, for $1 \leqslant p<d$, the Sobolev embedding theorem states that

$$
W^{1, p}\left(\mathbb{R}^{d}\right) \subset L^{q}\left(\mathbb{R}^{d}\right)
$$

for

$$
q=\frac{d p}{d-p}
$$

that is, $q$ is the Sobolev conjugate of $p$. Here $W^{1, p}\left(\mathbb{R}^{d}\right)$ is the Sobolev space consisting of real-valued functions $f$ on $\mathbb{R}^{d}$ such that both $f$ and all weak derivatives of $f$ are in $L^{p}\left(\mathbb{R}^{d}\right)$.

\section{A natural family of examples: proof of theorem 2.2}

We first show that $g_{t}$ is $\alpha$-Hölder, for

$$
\alpha=\frac{t p}{t+1} \wedge 1
$$

Let $0<x<y<1$ and let $y^{*} \in(0, y)$ be the largest value which satisfies $\arg \left(g_{t}\left(y^{*}\right)\right)=\arg$ $\left(g_{t}(y)\right)+\pi$. In order to prove that $g_{t}$ is $\alpha$-Hölder, it suffices to show

$$
\frac{\left|g_{t}(x)-g_{t}(y)\right|}{|x-y|^{\alpha}} \lesssim 1
$$

If $x<y^{*}$, then both $|x-y|>\left|y^{*}-y\right|$ and $\left|g_{t}(x)-g_{t}(y)\right|<\left|g_{t}\left(y^{*}\right)-g_{t}(y)\right|$ and hence it suffices to bound

$$
\sup _{y^{*} \leqslant x<y} \frac{\left|g_{t}(x)-g_{t}(y)\right|}{|x-y|^{\alpha}}=\sup _{y^{*} \leqslant x<y} \frac{\sqrt{x^{2 t p}+y^{2 t p}-2(x y)^{t p} \cos \left(x^{-t}-y^{-t}\right)}}{(y-x)^{\alpha}}
$$


from above by a constant independent of $y$. By Taylor's theorem $\cos z \geqslant 1-z^{2} / 2$ and applying this estimate to the function inside the square root in (3.1), we get

$$
x^{2 t p}+y^{2 t p}-2(x y)^{t p} \cos \left(x^{-t}-y^{-t}\right) \leqslant\left(y^{t p}-x^{t p}\right)^{2}+\left(y^{t}-x^{t}\right)^{2}(x y)^{t(p-2)}
$$

and therefore applying the inequality $\sqrt{a+b} \leqslant \sqrt{a}+\sqrt{b}$ for $a, b \geqslant 0$ in (3.1) this gives

$$
\sup _{y^{*} \leqslant x<y} \frac{\left|g_{t}(x)-g_{t}(y)\right|}{|x-y|^{\alpha}} \leqslant \sup _{y^{*} \leqslant x<y} \frac{\left(y^{t p}-x^{t p}\right)+\left(y^{t}-x^{t}\right)(x y)^{t(p / 2-1)}}{(y-x)^{\alpha}} .
$$

Considering only the first term, we have

$$
\sup _{y^{*} \leqslant x<y} \frac{y^{t p}-x^{t p}}{(y-x)^{\alpha}} \lesssim \sup _{y^{*} \leqslant x<y}(y-x)^{t p \wedge 1-\alpha} \leqslant 1
$$

For the remaining term, fix $x \in\left(y^{*}, y\right)$ and let $\omega=\omega(x, y) \in(0, \infty)$ be such that

$$
\arg \left(g_{t}(x)\right)=\arg \left(g_{t}(y)\right)+\pi y^{\omega} .
$$

Directly from the definition of $\omega$ we have

$$
\frac{y^{t}-x^{t}}{(x y)^{t}}=\pi y^{\omega}
$$

Moreover, this yields

$$
y-x=y-\frac{y}{\left(1+\pi y^{\omega+t}\right)^{1 / t}} \approx y\left(1+\pi y^{t+\omega}\right)^{1 / t}-y \approx y^{1+\omega+t}
$$

by Taylor's Theorem. This gives $x \geqslant y / 2$ for sufficiently small $y$ and so may use the estimate $x \approx y$ for all $x, y$ (with the implicit constants independent of $x$ and $y$ ). Therefore we have

$$
\frac{\left(y^{t}-x^{t}\right)(x y)^{t(p / 2-1)}}{(y-x)^{\alpha}} \approx y^{\omega} \frac{y^{t p}}{y^{(1+t+\omega) \alpha}} \leqslant 1
$$

since

$$
\alpha=\frac{t p}{t+1} \wedge 1 \leqslant \frac{t p+\omega}{t+1+\omega}
$$

for all $\omega>0$. Specifically, if $\alpha<1$, then the right-hand side is increasing in $\omega$ and so minimized at $\omega=0$, and if $\alpha=1$, then the right-hand side is uniformly bounded below by 1 . This proves that $g_{t}$ is $\alpha$-Hölder.

It remains to show $\alpha=t p /(t+1) \wedge 1$ is the sharp Hölder exponent, that is, $g_{t}$ is not $\alpha^{\prime}$ Hölder for $\alpha^{\prime} \in(\alpha, 1]$. Here we may assume that $\alpha=t p /(t+1)<1$, since otherwise there is nothing to prove. To this end, let $y \in(0,1)$ and choose $x=y^{*}$, and note that

$$
\frac{\left|g_{t}(x)-g_{t}(y)\right|}{|x-y|^{\alpha^{\prime}}}=\frac{x^{t p}+y^{t p}}{(y-x)^{\alpha^{\prime}}} .
$$

Observe that, as above, $x \approx y$ and $y-x \approx y^{1+t}$, noting that $\omega=\omega\left(y^{*}, y\right)=0$. Therefore

$$
\frac{\left|g_{t}(x)-g_{t}(y)\right|}{|x-y|^{\alpha^{\prime}}} \gtrsim y^{t p-(1+t) \alpha^{\prime}} \rightarrow \infty
$$


as $y \rightarrow 0$ if

$$
\alpha^{\prime}>\frac{t p}{t+1}
$$

proving the result.

Next we show that $g_{t}^{-1}$ is $\beta^{-1}$-Hölder, for $\beta=t p \vee 1$. It suffices to show that

$$
\frac{\left|g_{t}(x)-g_{t}(y)\right|}{|x-y|^{\beta}} \gtrsim 1
$$

with implicit constants independent of $x$ and $y$. Fix $0<x<y<1$ and, for $m \geqslant 1$, let $y_{m} \in(0, y)$ be the $m$ th largest number satisfying

$$
\arg \left(g_{t}\left(y_{m}\right)\right)=\arg \left(g_{t}(y)\right) .
$$

If $x \in\left[y_{m+1}, y_{m}\right)$ for $m \geqslant 1$, then $|x-y| \leqslant\left|y_{m+1}-y\right|$ and

$$
\left|g_{t}(x)-g_{t}(y)\right| \geqslant\left|g_{t}\left(y_{m}\right)-g_{t}(y)\right| \gtrsim\left|g_{t}\left(y_{m+1}\right)-g_{t}(y)\right|
$$

with implicit constant independent of $m$. In particular,

$$
\frac{\left|g_{t}(x)-g_{t}(y)\right|}{|x-y|^{\beta}} \gtrsim \frac{\left|g_{t}\left(y_{m+1}\right)-g_{t}(y)\right|}{\left|y_{m+1}-y\right|^{\beta}} .
$$

Recall the winding intervals $\mathcal{I}^{k}=g_{t}^{-1}\left(\mathcal{S}_{p}^{k}\right) \subset(0,1)$, now defined for $g_{t}$, see (2.1) and (2.2). In particular, $\mathcal{I}^{k}=\left[a_{k+1}, a_{k}\right)$ where

$$
\arg \left(g_{t}\left(a_{k}\right)\right)=a_{k}^{-t}=1+2 \pi(k-1)
$$

and therefore

$$
\left|\mathcal{I}^{k}\right| \approx(k-1)^{-1 / t}-k^{-1 / t} \approx k^{-1 / t-1} .
$$

If $y \in \mathcal{I}^{l}$ for some $l \geqslant 1$, then $y_{m+1} \in \mathcal{I}^{l+m+1}$ and so

$$
\begin{aligned}
\left|y_{m+1}-y\right|^{\beta} \lesssim\left(\sum_{k=l}^{l+m+1}\left|\mathcal{I}^{k}\right|\right)^{\beta} & \approx\left(\sum_{k=l}^{l+m+1} k^{-1 / t-1}\right)^{\beta} \\
& \approx\left(l^{-1 / t}-(l+m+1)^{-1 / t}\right)^{\beta} \\
& \leqslant l^{-p}-(l+m+1)^{-p}
\end{aligned}
$$

and

$$
\left|g_{t}\left(y_{m+1}\right)-g_{t}(y)\right| \approx \sum_{k=l}^{l+m+1}\left|g_{t}\left(y_{k+1}\right)-g_{t}\left(y_{k}\right)\right| \approx \sum_{k=l}^{l+m+1} k^{-p-1} \approx l^{-p}-(l+m+1)^{-p} .
$$

Therefore

$$
\frac{\left|g_{t}(x)-g_{t}(y)\right|}{|x-y|^{\beta}} \gtrsim 1
$$

Finally, suppose $x \in\left(y_{1}, y\right)$. If $x \in\left[y^{*}, y\right)$, where, as above, $y^{*} \in(0, y)$ is the largest value which satisfies $\arg \left(g_{t}\left(y^{*}\right)\right)=\arg \left(g_{t}(y)\right)+\pi$, then 


$$
\left|g_{t}(x)-g_{t}(y)\right| \gtrsim|x-y|^{\beta}
$$

If $x \in\left[y_{1}, y^{*}\right)$, and $y \in \mathcal{I}^{l}$ for some $l \geqslant 1$, then

$$
\frac{\left|g_{t}(x)-g_{t}(y)\right|}{|x-y|^{\beta}} \gtrsim \frac{\left|g_{t}\left(y_{1}\right)-g_{t}(y)\right|}{\left|y_{1}-y\right|^{\beta}} \gtrsim \frac{l^{-p-1}}{l^{-(1 / t+1) \beta}} \geqslant 1,
$$

since $\beta \geqslant t(p+1) /(t+1)$. This completes the proof that $g_{t}^{-1}$ is $\beta^{-1}$-Hölder.

The fact that $\beta^{-1}$ is the sharp Hölder exponent for $g_{t}^{-1}$ follows from theorem 2.1 since $\alpha$ and $\beta$ are 'winding conjugates', that is, they satisfy

$$
\beta=\frac{p \alpha}{p-\alpha}
$$

The proof of theorem 2.2 is complete.

\section{Hölder estimates from dimension theory}

If $g: X \rightarrow Y$ is an onto $\alpha$-Hölder map, then

$$
\operatorname{dim} Y \leqslant \frac{\operatorname{dim} X}{\alpha}
$$

where dim is the Hausdorff, packing, upper or lower box dimension, see $[\mathrm{F}]$. Moreover, if $g$ is Lipschitz, then

$$
\mathcal{H}^{1}(Y) \lesssim \mathcal{H}^{1}(X)
$$

where $\mathcal{H}^{1}$ is the one-dimensional Hausdorff measure. These estimates, and the analogous formulations for $g^{-1}$, give rise to bounds on $\alpha$ and $\beta$ in the Hölder winding problem. We consider these estimates in this section, ultimately proving that they are not sharp.

We refer the reader to [F, R, FY] for more background on dimension theory, including definitions and basic properties of the various dimensions. We recall the definitions of the box dimension and the Assouad spectrum here, which are the definitions we use directly. We omit the definition of $\mathcal{H}^{1}$ since the only properties we need are that it is a measure and that the $\mathcal{H}^{1}$ measure of the boundary of a circle is comparable to its radius.

Let $F \subseteq \mathbb{R}^{d}$ be a non-empty bounded set. The lower and upper box dimensions of $F$ are defined by

$$
\underline{\operatorname{dim}}_{\mathrm{B}} F=\liminf _{r \rightarrow 0} \frac{\log N_{r}(F)}{-\log r} \quad \text { and } \quad \overline{\operatorname{dim}}_{\mathrm{B}} F=\limsup _{r \rightarrow 0} \frac{\log N_{r}(F)}{-\log r}
$$

respectively, where $N_{r}(F)$ is the smallest number of sets required for an $r$-cover of $F$. If $\underline{\operatorname{dim}}_{\mathrm{B}} F=\overline{\operatorname{dim}}_{\mathrm{B}} F$, then we call the common value the box dimension of $F$ and denote it by $\operatorname{dim}_{\mathrm{B}} F$.

The Assouad spectrum of $F$ is defined as the function $\theta \mapsto \operatorname{dim}_{\mathrm{A}}^{\theta} F$ where

$$
\begin{aligned}
\operatorname{dim}_{\mathrm{A}}^{\theta} F= & \inf \{\alpha: \text { there exists } C>0 \text { such that, for all } 0<r<1 \text { and } x \in F, \\
& \left.N_{r}\left(B\left(x, r^{\theta}\right) \cap F\right) \leqslant C\left(\frac{r^{\theta}}{r}\right)^{\alpha}\right\} .
\end{aligned}
$$


and $\theta \in(0,1)$. This notion was introduced in [FY] and is similar in spirit to the Assouad dimension. The key difference is that the Assouad dimension considers all pairs of scales $r<R$, whereas here the parameter $\theta$ serves to fix the relationship between the big scale $R=r^{\theta}$ and the small scale $r$. The result is that the Assouad spectrum captures more precise information about the set, and has the benefit of being easier to work with and better behaved (see applications below). It also continuously interpolates between the upper box and (quasi-) Assouad dimension in a meaningful way. The quasi-Assouad dimension is another related notion which can be defined by

$$
\operatorname{dim}_{\mathrm{qA}} F=\lim _{\theta \rightarrow 1} \operatorname{dim}_{\mathrm{A}}^{\theta} F .
$$

Note that this is not the original definition of the quasi-Assouad dimension, see [LX], but this formula (and the fact the limit exists) was established in [FHHTY]. Also, the Assouad dimension, $\operatorname{dim}_{\mathrm{A}}$, which we will not use directly, satisfies $\operatorname{dim}_{\mathrm{qA}} F \operatorname{dim}_{\mathrm{A}} F \leqslant d$. Moreover, it was proved in $[\mathrm{FY}]$ that

$$
\overline{\operatorname{dim}}_{\mathrm{B}} F \leqslant \operatorname{dim}_{\mathrm{A}}^{\theta} F \leqslant \frac{\overline{\operatorname{dim}}_{\mathrm{B}} F}{1-\theta} \wedge \operatorname{dim}_{\mathrm{qA}} F
$$

and that $\operatorname{dim}_{\mathrm{A}}^{\theta} F$ is continuous in $\theta$.

We turn our attention now to the dimensions of spirals and the resulting applications to the winding problem. First, we note that the Hausdorff and packing dimensions of $\mathcal{S}_{p}$ are 1 for all $p>0$ and so no information can be gleaned from these dimensions since the dimensions of $(0,1)$ are also 1 . One can get some weak information by considering the length (one-dimensional Hausdorff measure) of $\mathcal{S}_{p}$ via the following simple result.

Theorem 4.1. If $p>1$ then $0<\mathcal{H}^{1}\left(\mathcal{S}_{p}\right)<\infty$, and if $p \leqslant 1$ then $\mathcal{H}^{1}\left(\mathcal{S}_{p}\right)=\infty$. Therefore, if $p \leqslant 1$, then there cannot exist an onto Lipschitz map $f:(0,1) \rightarrow \mathcal{S}_{p}$.

Proof. Clearly

$$
\mathcal{H}^{1}\left(\mathcal{S}_{p}^{k}\right) \approx k^{-p}
$$

and so

$$
\mathcal{H}^{1}\left(\mathcal{S}_{p}\right)=\sum_{k \geqslant 1} \mathcal{H}^{1}\left(\mathcal{S}_{p}^{k}\right) \approx \sum_{k \geqslant 1} k^{-p}
$$

from which the result follows.

Next we consider the box dimensions of $\mathcal{S}_{p}$. These are strictly greater than 1 for $p \in(0,1)$, which therefore improves on the information contained in the previous theorem concerning the winding problem. The following result can be found in [T, VH], but we include our own proof since it informs the strategy in the more complicated setting of the Assouad spectrum which follows. See also [ZZ] for a treatment of the box dimensions of spirals in $\mathbb{R}^{3}$.

Theorem 4.2. For all $p>0$

$$
\operatorname{dim}_{\mathrm{B}} \mathcal{S}_{p}=\frac{2}{1+p} \vee 1
$$

Proof. Let $r \in(0,1)$ and $k(r)$ be the unique positive integer satisfying

$$
k(r)^{-(p+1)} \leqslant r<(k(r)-1)^{-(p+1)},
$$


noting that $k(r) \approx r^{-1 /(p+1)}$. The importance of this parameter is that, decomposing $\mathcal{S}_{p}$ as the disjoint union of two sets

$$
\left(\bigcup_{k>k(r)} \mathcal{S}_{p}^{k}\right) \cup\left(\bigcup_{k \leqslant k(r)} \mathcal{S}_{p}^{k}\right),
$$

we see that $B\left(0, k(r)^{-p}\right)$ is contained in the $\delta$-neighbourhood of the first set for some $\delta \approx r$ since this portion of the spiral is wound 'tighter' than $\approx r$. However, a given $r$-ball may only cover part of second set with length $\lesssim r$, since the turns in the spiral are still ' $r$-separated' at this point. It follows that

$$
\begin{aligned}
N_{r}\left(\mathcal{S}_{p}\right) & \approx N_{r}\left(\mathcal{S}_{p} \cap B\left(0, k(r)^{-p}\right)\right)+\sum_{k=1}^{k(r)} N_{r}\left(\mathcal{S}_{p}^{k}\right) \\
& \approx\left(\frac{k(r)^{-p}}{r}\right)^{2}+\sum_{k=1}^{k(r)} \frac{k^{-p}}{r} \\
& \approx r^{-\frac{2}{1+p}}+r^{-1} \sum_{k=1}^{k(r)} k^{-p} .
\end{aligned}
$$

Therefore, if $p>1$, we get

$$
N_{r}\left(\mathcal{S}_{p}\right) \approx r^{-1}
$$

if $p=1$, we get

$$
N_{r}\left(\mathcal{S}_{p}\right) \approx r^{-1}+\log k(r) \approx r^{-1}(1+|\log r|)
$$

and, if $p<1$, we get

$$
N_{r}\left(\mathcal{S}_{p}\right) \approx r^{-\frac{2}{1+p}}+r^{-1} k(r)^{1-p} \approx r^{-\frac{2}{1+p}}
$$

The result follows.

Applying (4.1) for box dimension, we get the following corollary in the context of the winding problem.

Corollary 4.3. If $f:(0,1) \rightarrow \mathcal{S}_{p}$ is an onto $\alpha$-Hölder map, then

$$
\alpha \leqslant \frac{p+1}{2} \wedge 1
$$

It was proved in [FY, theorem 7.2] that for a large class of spirals $\mathcal{S}(\phi)$ (including the spirals $\mathcal{S}_{p}$ which we study) that, if $\overline{\operatorname{dim}}_{\mathrm{B}} \mathcal{S}(\phi)>1$, then the Assouad spectrum of $\mathcal{S}(\phi)$ is given by

$$
\operatorname{dim}_{\mathrm{A}}^{\theta} \mathcal{S}(\phi)=\frac{\overline{\operatorname{dim}}_{\mathrm{B}} \mathcal{S}(\phi)}{1-\theta} \wedge 2
$$

Note that this is then the general upper bound from (4.2). In particular, this result combined with theorem 4.2 yields the Assouad spectrum of $\mathcal{S}_{p}$ for $p<1$. However, for $p \geqslant 1, \overline{\operatorname{dim}}_{\mathrm{B}} \mathcal{S}_{p}=1$ and so the Assouad spectrum is not derivable from [FY]. We compute it here and, surprisingly, it is not given by the general upper bound from (4.2) for $p>1$ (see figure 3). 

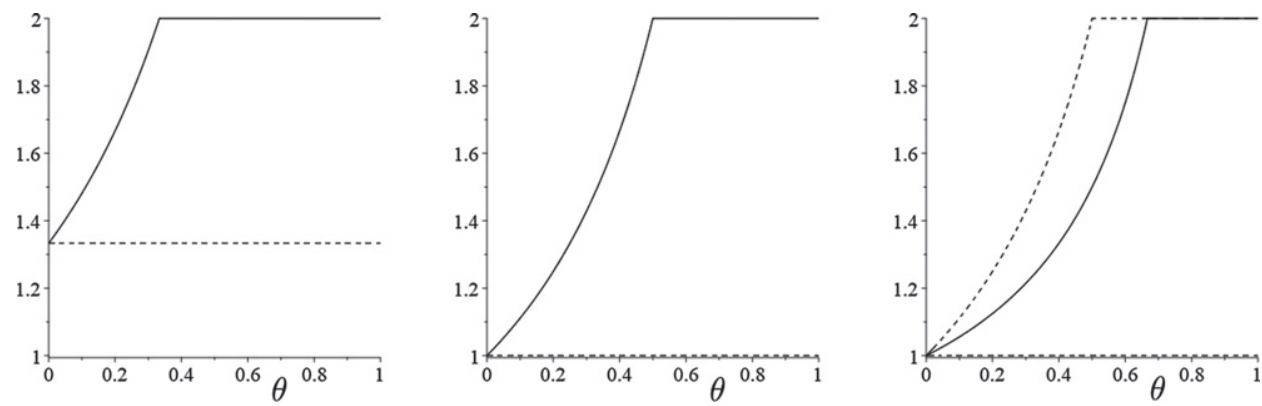

Figure 3. Plots of $\operatorname{dim}_{\mathrm{A}}^{\theta} \mathcal{S}_{p}$ for three different values of $p$. Moving from left to right, $p=1 / 2, p=1$, and $p=2$. The general upper and lower bounds from (4.2) are shown as dashed lines.

Theorem 4.4. For $p \in(0,1)$ and $\theta \in(0,1)$, we have

$$
\operatorname{dim}_{\mathrm{A}}^{\theta} \mathcal{S}_{p}=\frac{2}{(1+p)(1-\theta)} \wedge 2
$$

and, for $p \geqslant 1$ and $\theta \in(0,1)$, we have

$$
\operatorname{dim}_{\mathrm{A}}^{\theta} \mathcal{S}_{p}=\left(1+\frac{\theta}{p(1-\theta)}\right) \wedge 2 .
$$

In both cases, the Assouad spectrum has a single phase transition at $\theta=\frac{p}{1+p}$ and, if $p>1$, then the Assouad spectrum is strictly smaller than the upper bound from (4.2) for $0<\theta<\frac{p}{1+p}$.

Proof. The $p \in(0,1)$ case follows from theorem 4.2 and [FY, theorem 7.2], and therefore we assume $p \geqslant 1$. It suffices to prove the result for $0<\theta<\frac{p}{1+p}$, since for $\theta^{\prime}=\frac{p}{1+p}$ it follows by continuity of the Assouad spectrum that $\operatorname{dim}_{\mathrm{A}}^{\theta^{\prime}} \mathcal{S}_{p}=2$ and therefore by [FY, corollary 3.6] $\operatorname{dim}_{\mathrm{A}}^{\theta} \mathcal{S}_{p}=2$ for all $\theta>\frac{p}{1+p}$ as required. We prove the upper and lower bound separately, starting with the lower bound.

Let $r \in(0,1)$ and $l(r), L(r)$ be the unique positive integers satisfying

$$
L(r)^{-(p+1)} \leqslant r<(L(r)-1)^{-(p+1)}
$$

and

$$
l(r)^{-p} \leqslant r^{\theta}<(l(r)-1)^{-p},
$$

respectively. Note that

$$
L(r) \approx r^{-\frac{1}{p+1}} \quad \text { and } \quad l(r) \approx r^{-\frac{\theta}{p}}
$$

and so $L(r)>l(r)$ for all sufficiently small $r$ since we assume $\theta<\frac{p}{p+1}$. Arguing as in the proof of theorem 4.2 , we have

$$
\begin{aligned}
N_{r}\left(B\left(0, r^{\theta}\right) \cap \mathcal{S}_{p}\right) & \gtrsim \sum_{k=l(r)}^{L(r)} N_{r}\left(\mathcal{S}_{p}^{k}\right) \\
& \approx \sum_{k=l(r)}^{L(r)} \frac{k^{-p}}{r} .
\end{aligned}
$$


Therefore, if $p>1$ we get

$$
N_{r}\left(B\left(0, r^{\theta}\right) \cap \mathcal{S}_{p}\right) \gtrsim r^{-1}\left(l(r)^{1-p}-L(r)^{1-p}\right) \approx r^{-1-\frac{\theta(1-p)}{p}}=\left(\frac{r^{\theta}}{r}\right)^{1+\frac{\theta}{p(1-\theta)}}
$$

and if $p=1$ we get

$$
N_{r}\left(B\left(0, r^{\theta}\right) \cap \mathcal{S}_{p}\right) \gtrsim r^{-1}|\log r|=\left(\frac{r^{\theta}}{r}\right)^{\frac{1}{1-\theta}}|\log r|
$$

and in both cases we get the desired lower bound.

To prove the upper bound, we may assume that $p>1$ since the upper bound follows from (4.2) in the $p=1$ case. Let $r \in(0,1)$ and $l(r), L(r)$ be as before. We first deal with the case $z=0$. Once again arguing as in the proof of theorem 4.2, we have

$$
\begin{aligned}
N_{r}\left(B\left(0, r^{\theta}\right) \cap \mathcal{S}_{p}\right) & \lesssim N_{r}\left(B\left(0, L(r)^{-p}\right) \cap \mathcal{S}_{p}\right)+\sum_{k=l(r)}^{L(r)} N_{r}\left(\mathcal{S}_{p}^{k}\right) \\
& \approx\left(\frac{L(r)^{-p}}{r}\right)^{2}+\sum_{k=l(r)}^{L(r)} \frac{k^{-p}}{r} \\
& \lesssim\left(\frac{r^{\frac{p}{p+1}}}{r}\right)^{2}+r^{-1} l(r)^{1-p} \\
& \approx\left(\frac{r^{\theta}}{r}\right)^{\frac{2}{(1-\theta)(p+1)}}+\left(\frac{r^{\theta}}{r}\right)^{1+\frac{\theta}{p(1-\theta)}} .
\end{aligned}
$$

Finally we consider the case $z \neq 0$. If $|z| \leqslant 2 r^{\theta}$, then the above estimates hold up to uniform constants and so we assume $|z|>2 r^{\theta}$. We can make crude estimates in this case. If $k \geqslant 1$ is such that

$$
B\left(z, r^{\theta}\right) \cap \mathcal{S}_{p}^{k} \neq \emptyset,
$$

then $k \lesssim r^{-\theta / p}$ and $N_{r}\left(B\left(z, r^{\theta}\right) \cap \mathcal{S}_{p}^{k}\right) \lesssim r^{\theta} / r$. This gives

$$
N_{r}\left(B\left(z, r^{\theta}\right) \cap \mathcal{S}_{p}\right) \lesssim\left(r^{-\theta / p}\right)\left(r^{\theta} / r\right)=\left(\frac{r^{\theta}}{r}\right)^{1+\frac{\theta}{p(1-\theta)}} .
$$

We have proved

$$
\operatorname{dim}_{\mathrm{A}}^{\theta} \mathcal{S}_{p} \leqslant \frac{2}{(1-\theta)(p+1)} \vee\left(1+\frac{\theta}{p(1-\theta)}\right)=1+\frac{\theta}{p(1-\theta)}
$$

as required.

The following corollary follows from theorem 4.4. It was proved in the range $p \in(0,1)$ in [FY].

Corollary 4.5. For all $p>0, \operatorname{dim}_{\mathrm{A}} \mathcal{S}_{p}=\operatorname{dim}_{\mathrm{qA}} \mathcal{S}_{p}=2$.

The Assouad dimension does not behave well under Hölder image, see [LX], and so we cannot glean any information from knowledge of the Assouad dimension, despite it being large. 

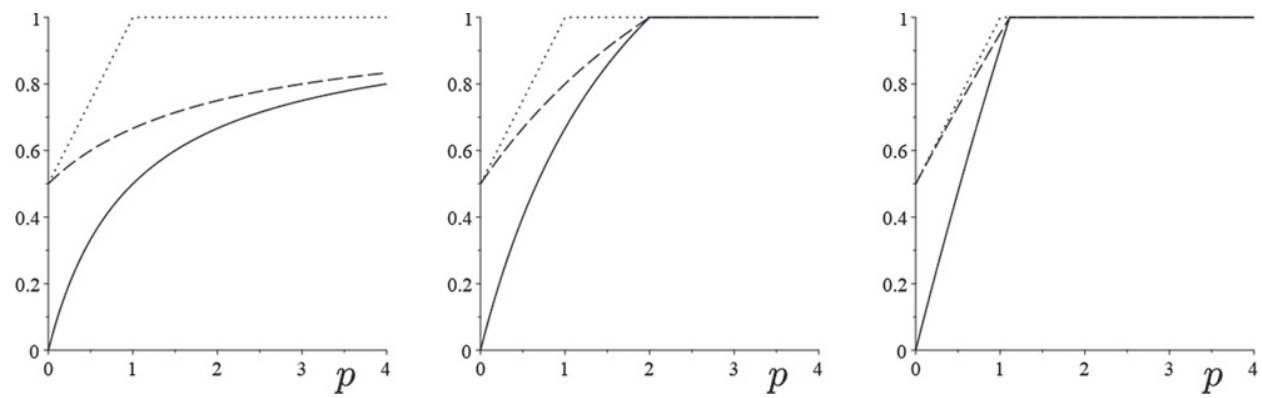

Figure 4. Upper bounds on $\alpha$, given the existence of an $(\alpha, \beta)$-Hölder homeomorphism between $(0,1)$ and $\mathcal{S}_{p}$. On the left, $\beta=1$, in the centre $\beta=2$, and on the right $\beta=10$. The sharp bounds are a solid line, the bounds obtained from the Assouad spectrum are a dashed line, and the bounds obtained from the box dimension are a dotted line.

However, the Assouad spectrum is more regular, and can be controlled in this context, however the control is more complicated than (4.1).

Lemma 4.6 (Theorem 4.11 [FY]). Let $X, Y \subseteq \mathbb{R}^{d}$ and $0<\alpha \leqslant 1 \leqslant \beta<\infty$. If $g: X \rightarrow Y$ is an $(\alpha, \beta)$-Hölder homeomorphism, then

$$
\operatorname{dim}_{\mathrm{A}} Y \geqslant \frac{\operatorname{dim}_{\mathrm{A}} X\left(1-\theta_{0}\right)}{\beta-\alpha \theta_{0}},
$$

where $\theta_{0}=\inf \left\{\theta \in(0,1): \operatorname{dim}_{\mathrm{A}}^{\theta} X=\operatorname{dim}_{\mathrm{A}} X\right\}$. For convenience we write $\inf \emptyset=1$.

Applying lemma 4.6 with $g=f^{-1}$ we obtain the following bounds.

Corollary 4.7. If $f:(0,1) \rightarrow \mathcal{S}_{p}$ is an $(\alpha, \beta)$-Hölder homeomorphism, then

$$
\beta \geqslant \frac{p \alpha}{1+p-2 \alpha} \vee 1 \text {. }
$$

Proof. Noting that $g=f^{-1}$ is a $\left(\beta^{-1}, \alpha^{-1}\right)$-Hölder homeomorphism between $\mathcal{S}_{p}$ and $(0,1)$, and

$$
\theta_{0}=\inf \left\{\theta \in(0,1): \operatorname{dim}_{\mathrm{A}}^{\theta} \mathcal{S}_{p}=\operatorname{dim}_{\mathrm{A}} \mathcal{S}_{p}\right\}=\frac{p}{p+1}
$$

we obtain

$$
1 \geqslant \frac{2\left(1-\frac{p}{p+1}\right)}{\alpha^{-1}-\beta^{-1} \frac{p}{p+1}}
$$

directly from lemma 4.6. Rearranging this formula for $\beta$ yields the desired bound, recalling that $\beta \geqslant 1$ is trivial.

For comparison, and in order to give an alternative expression of the bounds in terms of $\alpha$, we summarize the various estimates obtained so far in the following corollary (see figure 4).

Corollary 4.8. Suppose there exists an $(\alpha, \beta)$-Hölder homeomorphism between $(0,1)$ and $\mathcal{S}_{p}$. Then

$$
\alpha \leqslant \frac{p \beta}{p+\beta} \wedge 1
$$


and this bound is sharp. Based on knowledge of the Assouad spectrum, we have

$$
\alpha \leqslant \frac{p \beta+\beta}{p+2 \beta} \wedge 1,
$$

which is not sharp, and based on knowledge of the box dimension, we have

$$
\alpha \leqslant \frac{p+1}{2} \wedge 1,
$$

which is also not sharp.

Notice that as we relax the restrictions on the inverse map, that is, we let $\beta \rightarrow \infty$, the bounds obtained from the Assouad spectrum approach those obtained from the box dimension, and the sharp bounds approach those from the first part of theorem 2.1 which considers the forward Hölder exponent in isolation.

\section{An alternative proof of corollary 2.3}

In this section we provide an alternative proof of corollary 2.3 where, instead of considering a natural family of explicit examples, we directly construct a function with the desired properties. We decided to include both proofs for the interested reader. Moreover, we find the proof via theorem 2.2 more natural and appropriate in this setting, but the proof presented here is less reliant on the precise setting of the problem and may be more straightforward to generalize. Certain details in the proof will be similar to those from the proof of theorem 2.2 and will be suppressed. steps.

Fix $p>0$ and $\alpha \in\left[\frac{p}{p+1}, p\right) \cap(0,1]$. We construct a homeomorphism $f:(0,1) \rightarrow \mathcal{S}_{p}$ in four

Step 1 Partition the interval $(0,1)$ into countably many half open intervals $\mathcal{J}^{k}=[a, b)$ $(k \geqslant 1)$, labelled from right to left, which satisfy

$$
\left|\mathcal{J}^{k}\right| \approx k^{-p / \alpha}
$$

where the implicit constants are independent of $k$. This can be done since $\sum_{k \geqslant 1} k^{-p / \alpha} \approx 1$. These intervals will play the role of $\mathcal{I}^{k}$ for the function $f$, see (2.2).

Step 2 For $k \geqslant 1$, let $g_{0}^{k}: \mathcal{J}^{k} \rightarrow\left[0,\left|\mathcal{J}^{k}\right|^{\alpha}\right)$ be defined by

$$
g_{0}^{k}(x)=\left|\mathcal{J}^{k}\right|^{\alpha}-\left(\sup \mathcal{J}^{k}-x\right)^{\alpha} .
$$

In particular, $g_{0}^{k}$ is $(\alpha, 1)$-Hölder, with implicit constants independent of $k$.

Step 3 For $k \geqslant 1$, let $g_{1}^{k}:\left[0,\left|\mathcal{J}^{k}\right|^{\alpha}\right) \rightarrow \mathcal{S}_{p}^{k}$ be a smooth homeomorphism satisfying

$$
\mathcal{H}^{1}\left(g_{1}^{k}(J)\right) \approx|J|
$$

for all open intervals $J \subset\left[0,\left|\mathcal{J}^{k}\right|^{\alpha}\right.$ ), where the implicit constants are independent of $k$ and $J$. Such a map exists because $\mathcal{H}^{1}\left(\mathcal{S}_{p}^{k}\right) \approx k^{-p} \approx\left|\mathcal{J}^{k}\right|^{\alpha}$. Recall that $\mathcal{S}_{p}^{k}$ is the $k$ th full turn, see (2.1), and that $\mathcal{H}^{1}$ is the one-dimensional Hausdorff measure.

Step 4 Let $f:(0,1) \rightarrow \mathcal{S}_{p}$ be defined by

$$
\left.f\right|_{\mathcal{J}^{k}}(x)=g_{1}^{k} \circ g_{0}^{k}(x) .
$$


By construction, $f$ is a homeomorphism between $(0,1)$ and $\mathcal{S}_{p}$. Note that the maps $\left(g_{1}^{k} \circ g_{0}^{k}\right)_{k}$ are compatible at the endpoints since the intervals $\mathcal{J}^{k}$ were chosen to be half open. It remains to establish the Hölder exponents, which we separate into two claims.

Claim 1. $f$ is $\alpha$-Hölder.

Proof of Claim 1. Let $0<x<y<1$ and, as before, $y^{*} \in(0, y)$ be the largest value satisfying

$$
\arg \left(f\left(y^{*}\right)\right)=\arg (f(y))+\pi
$$

As in the proof of theorem 2.2, it suffices to prove

$$
\sup _{y^{*}<x<y} \frac{|f(x)-f(y)|}{|x-y|^{\alpha}} \lesssim 1
$$

where the implicit constant is independent of $y$. However, this follows immediately since $\left(y^{*}, y\right)$ can intersect at most 2 of the intervals $\mathcal{J}^{k}$. This relies on the fact that the maps $g_{1}^{k}$ are Lipschitz and the maps $g_{0}^{k}$ are $\alpha$-Hölder, both with implicit constants independent of $k$.

Claim 2. $f^{-1}$ is $\left(\frac{p-\alpha}{p \alpha}\right)$-Hölder.

Proof of Claim 2. Let $\beta=\frac{p \alpha}{p-\alpha}$ and $0<x<y<1$. Similar to above, for $m \geqslant 1$, let $y_{m} \in(0, y)$ be the $m$ th largest number satisfying

$$
\arg \left(f\left(y_{m}\right)\right)=\arg (f(y))
$$

If $x \in\left[y_{m+1}, y_{m}\right)$ for $m \geqslant 1$, then $|x-y| \leqslant\left|y_{m+1}-y\right|$ and

$$
|f(x)-f(y)| \geqslant\left|f\left(y_{m}\right)-f(y)\right| \gtrsim\left|f\left(y_{m+1}\right)-f(y)\right|
$$

with implicit constant independent of $m$. In particular,

$$
\frac{|f(x)-f(y)|}{|x-y|^{\beta}} \gtrsim \frac{\left|f\left(y_{m+1}\right)-f(y)\right|}{\left|y_{m+1}-y\right|^{\beta}} .
$$

If $y \in \mathcal{J}^{l}$ for some $l \geqslant 1$, then $y_{m+1} \in \mathcal{J}^{l+m+1}$ and so

$$
\begin{aligned}
\left|y_{m+1}-y\right|^{\beta} \lesssim\left(\sum_{k=l}^{l+m+1}\left|\mathcal{J}^{k}\right|\right)^{\beta} & \approx\left(\sum_{k=l}^{l+m+1} k^{-p / \alpha}\right)^{\beta} \\
& \approx\left(l^{1-p / \alpha}-(l+m+1)^{1-p / \alpha}\right)^{\beta} \\
& \leqslant l^{\beta(1-p / \alpha)}-(l+m+1)^{\beta(1-p / \alpha)} \\
& =l^{-p}-(l+m+1)^{-p}
\end{aligned}
$$

and

$$
\left|f\left(y_{m+1}\right)-f(y)\right| \approx \sum_{k=l}^{l+m+1} k^{-p-1} \approx l^{-p}-(l+m+1)^{-p} .
$$


Therefore

$$
\frac{|f(x)-f(y)|}{|x-y|^{\beta}} \gtrsim 1
$$

as required.

Finally, suppose $x \in\left(y_{1}, y\right)$. If $x \in\left[y^{*}, y\right)$, where $y^{*}$ is as above, then

$$
|f(x)-f(y)| \gtrsim|x-y|
$$

since $\left(y^{*}, y\right)$ can intersect at most 2 of the intervals $\mathcal{J}^{k}$. This relies on the fact that the maps $g_{1}^{k}$ are bi-Lipschitz on the interval $\left(y^{*}, y\right)$ since it only corresponds to a half turn in $\mathcal{S}_{p}$, and the maps $g_{0}^{k}$ are $(\alpha, 1)$-Hölder. If $x \in\left[y_{1}, y^{*}\right)$, and $y \in \mathcal{J}^{l}$ for some $l \geqslant 1$, then

$$
\frac{|f(x)-f(y)|}{|x-y|^{\beta}} \gtrsim \frac{\left|f\left(y_{1}\right)-f(y)\right|}{\left|y_{1}-y\right|^{\beta}} \gtrsim \frac{l^{-p-1}}{l^{-\beta p / \alpha}} \gtrsim 1
$$

completing the proof. Note that the final bound relies on the assumption that $\alpha \geqslant p /(p+1)$.

\section{Reduction to bi-Lipschitz classes}

In this section we prove a simple equivalence which extends our results to a much broader class of functions, as well as providing an example showing that our results do not generally hold in a slightly broader class still.

Theorem 6.1. Let $\phi$ be a winding function such that the function $\varepsilon:[1, \infty) \rightarrow(0, \infty)$ defined by

$$
\varepsilon(x)=\phi(x) x^{p}
$$

is Lipschitz and uniformly bounded away from 0 and $\infty$. Then $\mathcal{S}_{p}$ and $\mathcal{S}(\phi)$ are bi-Lipschitz equivalent, that is, there is a bi-Lipschitz homeomorphism between $\mathcal{S}_{p}$ and $\mathcal{S}(\phi)$.

Proof. Since $\varepsilon$ is Lipschitz, there exists a constant $L>0$ such that

$$
|\varepsilon(x)-\varepsilon(x)| \leqslant L|x-y|
$$

for all $x, y \geqslant 1$. Let $F: \mathcal{S}_{p} \rightarrow \mathcal{S}(\phi)$ be defined by

$$
F\left(x^{-p} \exp (\mathrm{i} x)\right)=\phi(x) \exp (\mathrm{i} x)
$$

and consider points $x>y \geqslant 1$. If $x-y(\bmod 2 \pi) \in(\pi / 2,3 \pi / 2)$, then we immediately get

$$
\frac{|\phi(x) \exp (\mathrm{i} x)-\phi(y) \exp (\mathrm{i} y)|}{\left|x^{-p} \exp (\mathrm{i} x)-y^{-p} \exp (\mathrm{i} y)\right|} \approx \frac{\phi(y)}{y^{-p}}=\varepsilon(y) \approx 1 .
$$

If $x-y(\bmod 2 \pi) \notin(\pi / 2,3 \pi / 2)$, then a slightly more complicated argument is needed. Applying the bounds $1-z^{2} / 2 \leqslant \cos z \leqslant 1-z^{2} / 3(|z| \leqslant \pi / 2)$ we get 


$$
\begin{aligned}
\frac{|\phi(x) \exp (\mathrm{i} x)-\phi(y) \exp (\mathrm{i} y)|}{\left|x^{-p} \exp (\mathrm{i} x)-y^{-p} \exp (\mathrm{i} y)\right|} & =\frac{\sqrt{\phi(x)^{2}+\phi(y)^{2}-2 \phi(x) \phi(y) \cos (x-y)}}{\sqrt{x^{-2 p}+y^{-2 p}-2 x^{-p} y^{-p} \cos (x-y)}} \\
& \leqslant \frac{\sqrt{(\phi(y)-\phi(x))^{2}+\phi(x) \phi(y)(x-y)^{2}}}{\sqrt{\left(y^{-p}-x^{-p}\right)^{2}+\frac{2}{3} x^{-p} y^{-p}(x-y)^{2}}} \\
& \lesssim \frac{\sqrt{\left(y^{-p} \varepsilon(y)-x^{-p} \varepsilon(x)\right)^{2}+x^{-p} y^{-p}(x-y)^{2}}}{\sqrt{\left(y^{-p}-x^{-p}\right)^{2}+x^{-p} y^{-p}(x-y)^{2}}} .
\end{aligned}
$$

Using the fact that $\varepsilon(x) \geqslant \varepsilon(y)-L(x-y)$ we get

$$
\begin{aligned}
\left(y^{-p} \varepsilon(y)-x^{-p} \varepsilon(x)\right)^{2} \leqslant & \left(y^{-p} \varepsilon(y)-x^{-p}(\varepsilon(y)-L(x-y))\right)^{2} \\
= & \varepsilon(y)^{2}\left(y^{-p}-x^{-p}\right)^{2}+2 L x^{-p} \varepsilon(y)\left(y^{-p}-x^{-p}\right)(x-y) \\
& \quad+L^{2} x^{-2 p}(x-y)^{2} \\
\lesssim & \left(y^{-p}-x^{-p}\right)^{2}+x^{-p}\left(y^{-p}-x^{-p}\right)(x-y) \\
& \quad+x^{-p} y^{-p}(x-y)^{2} \\
\lesssim & \left(y^{-p}-x^{-p}\right)^{2}+x^{-p} y^{-p}(x-y)^{2}
\end{aligned}
$$

Here the final line follows since the middle of the three terms in the previous line is bounded above by the maximum of the other two. This proves that $(6.1)$ is $\lesssim 1$, proving that $F$ is Lipschitz. The proof that $(6.1)$ is also $\gtrsim 1$ is similar and omitted and establishes that $F$ is bi-Lipschitz as required.

An immediate consequence of theorem 6.1 is that we can replace $\mathcal{S}_{p}$ with $\mathcal{S}(\phi)$ in all of our main results in this paper where $\phi$ is any winding function such that $\phi(x) x^{p}$ is Lipschitz and uniformly bounded away from 0 and $\infty$. In particular, in theorem 2.1, and corollary 2.3, as well as the dimension results in theorems 4.2 and 4.4. For example, $\phi(x)$ can be the reciprocal of any polynomial of degree $p$ which is strictly increasing on $[1, \infty)$. More complicated functions also work, including many non-differentiable functions or non-polynomial functions, such as

$$
\phi(x)=\frac{3}{5 x^{p} x^{-1 / x}+x^{p / 2} \log (x)}
$$

which is comparable to $x^{-p}$ in the above sense.

It would be interesting to push theorem 6.1 further, with the most natural class to consider being $\phi$ such that $\phi(x) x^{p}$ is not uniformly bounded away from 0 and $\infty$, but can be controlled by a lower order function, such as $\log (x)$. For example, are $\mathcal{S}_{p}$ and $\mathcal{S}(\phi)$ bi-Lipschitz equivalent for $\phi(x)=x^{-p} \log x$ ? In fact this turns out to be false, which we show by adapting the arguments from theorem 2.1. In the following result, compare the strict lower bound for $\beta$ with the corresponding bound from theorem 2.1.

Theorem 6.2. Let $p, \gamma>0$ and $\phi(x)=x^{-p}(\log x)^{\gamma}$. If $f:(0,1) \rightarrow \mathcal{S}(\phi)$ is an $(\alpha, \beta)$-Hölder homeomorphism, then $\alpha<p$ and

$$
\beta>\frac{p \alpha}{p-\alpha}
$$


Proof. Analogous to (2.1) and (2.2), let

$$
\mathcal{S}^{k}=\{\phi(x) \exp (\mathrm{i} x): 1+2 \pi(k-1)<x \leqslant 1+2 \pi k\}
$$

for integer $k \geqslant 1$ and $\mathcal{I}^{k}=f^{-1}\left(\mathcal{S}^{k}\right)$. We have

$$
k^{-p}(\log k)^{\gamma} \approx\left|\mathcal{S}^{k}\right|=\left|f\left(\mathcal{I}^{k}\right)\right| \lesssim\left|\mathcal{I}^{k}\right|^{\alpha}
$$

and therefore

$$
1=\sum_{k=1}^{\infty}\left|\mathcal{I}^{k}\right| \gtrsim \sum_{k=1}^{\infty} k^{-p / \alpha}(\log k)^{\gamma / \alpha}
$$

which forces $\alpha<p$. Suppose

$$
\beta \leqslant \frac{p \alpha}{p-\alpha}
$$

and, for integer $l \geqslant 1$, let

$$
x_{l}=\sum_{k=l}^{\infty}\left|\mathcal{I}^{k}\right| \text {. }
$$

Extending $f$ continuously to $[0,1]$ and applying (6.2) yields

$$
\begin{aligned}
1 \lesssim & \frac{\left|f\left(x_{l}\right)-f(0)\right|}{\left|x_{l}\right|^{\beta}} \lesssim \frac{l^{-p}(\log l)^{\gamma}}{\left(\sum_{k=l}^{\infty}\left|\mathcal{I}^{k}\right|\right)^{\beta}} \lesssim \frac{l^{-p}(\log l)^{\gamma}}{\left(\sum_{k=l}^{\infty} k^{-p / \alpha}(\log k)^{\gamma / \alpha}\right)^{\beta}} \\
& \lesssim \frac{l^{-p}(\log l)^{\gamma}}{l^{\beta(1-p / \alpha)}(\log l)^{\beta \gamma / \alpha}} \rightarrow 0,
\end{aligned}
$$

a contradiction. To see the final convergence, note that the polynomial part will tend to 0 , dominating the logarithmic part, unless $\beta=p \alpha /(p-\alpha)$ in which case the polynomial part disappears. However, in this case $\beta / \alpha>1$ and the logarithmic part tends to 0 .

Corollary 6.3. Let $p, \gamma>0$ and $\phi(x)=x^{-p}(\log x)^{\gamma}$. Then the spirals $\mathcal{S}_{p}$ and $\mathcal{S}(\phi)$ are not bi-Lipschitz equivalent.

Proof. This follows immediately from theorem 6.2 since it is not possible to choose $\alpha=\beta=1$.

\section{Acknowledgments}

The author was supported by an EPSRC Standard Grant No. (EP/R015104/1) and a Leverhulme Trust Research Project Grant No. (RPG-2019-034). He thanks Han Yu for several stimulating conversations on spiral winding and the Assouad spectrum, and David Dritschel for a helpful explanation of spiral formation in the context of $\alpha$-models for fluid turbulence. Finally, the author thanks an anonymous referee for carefully reading the paper and making several suggestions.

\section{ORCID iDs}

Jonathan M Fraser (iD) https://orcid.org/0000-0002-8066-9120 


\section{References}

Dupain Y, Mendès France M and Tricot C 1983 Dimensions des spirales Bul. Soc. Math. France 79 193-201

Falconer K J 2014 Fractal Geometry: Mathematical Foundations and Applications 3rd edn (New York: Wiley)

Foias C, Holm D D and Titi E S 2001 The Navier-Stokes-alpha model of fluid turbulence Physica D 152-153 505-19

Fish A and Paunescu L 2018 Unwinding spirals I Methods Appl. Anal. 25 225-32

Fraser J M, Hare K E, Hare K G, Troscheit S and Yu H 2019 The Assouad spectrum and the quasi-Assouad dimension: a tale of two spectra Ann. Acad. Sci. Fenn. Math. 44 379-87

Fraser J M and Yu H 2018 New dimension spectra: finer information on scaling and homogeneity Adv. Math. 329 273-328

Katznelson Y, Nag S and Sullivan D P 1990 On conformal welding homeomorphisms associated to Jordan curves Ann. Acad. Sci. Fenn. Math. 15 293-306

Lü F and Xi L-F 2016 Quasi-Assouad dimension of fractals J. Fractal Geometry 3 187-215

Mandelbrot B B 1982 The Fractal Geometry of Nature (San Francisco, CA: Freeman)

Moffatt H K 1993 Spiral structures in turbulent flow Wavelets, Fractals, and Fourier Transforms (The Institute of Mathematics and its Applications Conference Series vol 43) (New York: Oxford University Press) pp 317-24

Robinson J C 2011 Dimensions, Embeddings, and Attractors (Cambridge: Cambridge University Press)

Tricot C 1995 Curves and Fractal Dimension (New York: Springer) Translated from the 1993 French original

Vassilicos J C 1993 Fractals in turbulence Wavelets, Fractals, and Fourier Transforms (The Institute of Mathematics and its Applications Conference Series vol 43) (New York: Oxford University Press) pp 325-40

Vassilicos J C and Hunt J C R 1991 Fractal dimensions and spectra of interfaces with application to turbulence Proc. R. Soc. A 435 505-34

Žubrinić D and Županović V 2005 Box dimension of spiral trajectories of some vector fields in $\mathbb{R}^{3}$ Qual. Theory Dyn. Syst. 6 251-72 